\title{
Optimality Inequalities for Average Cost MDPs and their Inventory Control Applications
}

\author{
Eugene A. Feinberg
}

\author{
Mark E. Lewis
}

\begin{abstract}
We show that the assumptions guaranteeing existence of a solution to the average cost optimality inequalities presented in Schäl [1] for compact action sets can be extended to include the non-compact action set case. Inventory and stochastic cash balance models (with fixed costs) are natural candidates for the application of our results. Extension of the classic models for a general demand distribution are discussed in detail.
\end{abstract}

\section{INTRODUCTION}

In a discrete-time Markov decision process the usual method to study the average cost criterion is to find a solution to the average cost optimality equations. A policy that achieves the minimum in this system of equations is then average cost optimal. When the state and action spaces are infinite, one may be required to replace the equations with inequalities, yet the conclusions are the same; a policy that achieves the minimum in the inequalities is average cost optimal. Schäl [1] provides two sets of general conditions that imply the existence of a solution to the average cost optimality inequalities (ACOI). The first, referred to as Assumptions (W) in Schäl [1], require weak continuity of the transition probabilities. The second group, Assumptions (S), require setwise continuity of the transition probabilities. The purpose of this paper is to relax the assumptions in Schäl [1] so that the results can be applied directly to several problems in the literature; in particular to those related to inventory control.

Recall the typical dynamic state equation for inventory control models

$$
x_{n+1}=x_{n}+a_{n}-D_{n+1}, \quad n=0,1,2, \ldots,
$$

where $x_{n}$ is the inventory at the end of period $n, a_{n}$ is the decision how much should be ordered, and $D_{n}$ is the demand during period $n$. Let $q(d y \mid x, a)$ be the transition probability for the control problem (I.1). Weak continuity of $q$ means that $E_{x_{n}^{k}, a_{n}^{k}} f\left(x_{n+1}\right) \rightarrow E_{x_{n}, a_{n}} f\left(x_{n+1}\right)$ for any sequence $\left\{\left(x_{n}^{k}, a_{n}^{k}\right), k \geq 0\right\}$ such that $\left(x_{n}^{k}, a_{n}^{k}\right) \rightarrow$

Feinberg: Department of Applied Mathematics and Statistics, State University of New York at Stony Brook, Stony Brook, NY 11794-3600 (efeinberg@notes.cc.sunysb.edu)

Lewis: Department of Industrial and Operations Engineering, University of Michigan, 1205 Beal Avenue, Ann Arbor, MI 48109-2117 (melewis@engin.umich.edu) $\left(x_{n}, a_{n}\right)$ where $f$ is any bounded, continuous function and the expectation is taken with respect to $q$. This holds in most inventory applications in light of (I.1) and Lebesgue's dominated convergence theorem. On the other hand, setwise continuity is too strong. Recall that this means that $q\left(B \mid x_{n}^{k}, a_{n}^{k}\right) \rightarrow q\left(B \mid x_{n}, a_{n}\right)$ as $\left(x_{n}^{k}, a_{n}^{k}\right) \rightarrow\left(x_{n}, a_{n}\right)$ for any Borel set $B$. For example, let $D_{n}=1$ (deterministically), $a_{n}^{k}=a_{n}+\frac{1}{k}$ and $x_{n}^{k}=x_{n}$. Then $q\left(B \mid x_{n}, a_{n}\right)=1$ for $B=\left(-\infty, x_{n}+a_{n}-1\right]$ and $q\left(B \mid x_{n}, a_{n}^{k}\right)=0$ for all $k=1,2, \ldots$.

Unfortunately, under Assumptions (W) or (S) the results of Schäl [1] require that the action sets are compact. This prevents us from applying them directly since in many inventory control models the action sets are assumed unbounded. In this paper we discuss conditions, under which the compact action set assumption of Schäl [1] can be relaxed.

The inventory control literature is far too expansive to attempt a complete literature review. The reader is pointed to the survey article by Porteus [2]. In the case of inventory control, under the average cost criterion the optimality of $(s, S)$ policies was proved by Iglehart [3] and Veinott and Wagner [4] in the continuous and discrete demand cases, respectively. The latter proof was simplified significantly by Zheng [5]. A more recent paper in average cost inventory models is the work of Beyer and Sethi [6]. The authors reconsider the continuous demand model of Iglehart [3] and verify several assumptions that apparently were not stated in the original work. They also make the observation that most of the work following Iglehart's paper showing that $(s, S)$ policies are optimal in the average cost case assume some bounds on the inventory position after ordering. In the present work we require no such restriction.

Since our results hold for general Markov decision processes (MDPs) (inventory control is just an example) we point the reader to several excellent texts; Sennott [7] and Hernandez-Lerma and Lasserre [8] for countable and general state spaces, respectively. Hernández-Lerma and Lasserre [8], Chapter 5, and Fernández-Gaucherand [9] present results for non-compact action sets but assume setwise convergence. Moreover, Section 5.7 in HernándezLerma and Lasserre [8] provides conditions for the existence of stationary optimal policies for an MDP with weakly continuous transition probabilities but the derivation is 
done directly; without deriving the optimality equations or inequalities.

The rest of the paper is organized as follows. In Section II we discuss the general Markov decision process framework. Section III explains some related results from HernándezLerma and Lasserre [8] and Schäl [1]. Section IV contains the main theoretical contributions of the paper; two sets of assumptions that lead to the convergence of discounted cost optimal values and policies. We formulate the inventory and stochastic cash balance problems in Section V. In this same section, we show that these inventory problems satisfy the assumptions of Section IV. The paper is concluded in Section VI.

\section{MODEL DEFINITION}

Consider a discrete-time Markov decision process with state space $\mathbb{X}$ and action space $\mathbb{A}$. Assume that both $\mathbb{X}$ and $\mathbb{A}$ are Borel subsets of Polish (complete, separable, metric) spaces. For each $x \in \mathbb{X}$ the nonempty Borel subset $A(x)$ represents the set of actions available at $x$. Define the graph of $\mathbb{A}$ by

$$
\operatorname{Gr}(\mathbb{A}):=\{(x, a) \mid x \in \mathbb{X}, a \in A(x)\},
$$

and assume that: (i) $\operatorname{Gr}(\mathbb{A})$ is a measurable subset of $\mathbb{X} \times \mathbb{A}$, and (ii) there exists a measurable mapping $\phi$ from $\mathbb{X}$ to $\mathbb{A}$ such that $\phi(x) \in A(x)$ for all $x \in \mathbb{X}$. The one step cost, $c(x, a)$, for choosing action $a \in A(x)$ in state $x$ is presumed a non-negative (or equivalently, bounded below), measurable function on $\operatorname{Gr}(\mathbb{A})$. Let $q(B \mid x, a)$, also measurable on $\operatorname{Gr}(\mathbb{A})$, be the transition kernel representing the probability that $B \subseteq \mathbb{X}$ is entered next, given that action $a$ is chosen in state $x$. This means that $q(\cdot \mid x, a)$ is a probability measure on $\mathbb{X}$ for each pair $(x, a) \in \mathbb{X} \times \mathbb{A}$, and $q(B \mid \cdot, \cdot)$ is a Borel function on $\mathbb{X} \times \mathbb{A}$ for any Borel subset $B \subseteq \mathbb{X}$.

The decision process proceeds as follows: at time $n$ the current state of the system, $x$, is observed. A decisionmaker decides which action, $a$, to choose, the cost $c(x, a)$ is accrued, the system moves to the next state according to $q(\cdot \mid x, a)$, and the process continues. Let $H_{n}=(\mathbb{X} \times$ $\mathbb{A})^{n} \times \mathbb{X}$ be the set of histories for $n=0,1, \ldots$ A (randomized) decision rule at epoch $n=0,1, \ldots$ is a regular transition probability $\pi_{n}$ from $H_{n}$ to $\mathbb{A}$ concentrated on $A\left(x_{n}\right)$. In other words, (i) $\pi_{n}\left(\cdot \mid h_{n}\right)$ is a probability distribution on $\mathbb{A}$ such that $\pi_{n}\left(A\left(x_{n}\right) \mid h_{n}\right)=1$, where $h_{n}=\left(x_{0}, a_{0}, x_{1}, \ldots, a_{n-1}, x_{n}\right)$ and (ii) for any measurable subset $B \subseteq \mathbb{A}$, the function $\pi_{n}(B \mid \cdot)$ is measurable on $H_{n}$. A policy $\pi$ is a sequence $\left(\pi_{0}, \pi_{1}, \ldots\right)$ of decision rules. Moreover, $\pi$ is called non-randomized if each probability measure $\pi_{n}\left(\cdot \mid h_{n}\right)$ is concentrated at one point. A policy is called stationary if it is non-randomized and all decisions depend only on the current state. In other words, a stationary policy $\phi$ is defined by a measurable mapping $\phi: \mathbb{X} \rightarrow \mathbb{A}$ such that $\phi(x) \in A(x)$ for all $x \in \mathbb{X}$.
The Ionescu-Tulcea theorem (cf. p. 140-141 of [10] or p. 178 of [8]) yields that an initial state $x$ and a policy $\pi$ define a unique probability distribution $\mathbb{P}_{x}^{\pi}$ on the set of all trajectories $H_{\infty}=(\mathbb{X} \times \mathbb{A})^{\infty}$ endowed with the product $\sigma$-field defined by Borel $\sigma$-fields of $\mathbb{X}$ and $\mathbb{A}$. Let $\mathbb{E}_{x}^{\pi}$ be the expectation with respect to this distribution. Define

$$
\begin{aligned}
v_{\alpha}^{\pi}(x) & :=\lim _{N \rightarrow \infty} \mathbb{E}_{x}^{\pi} \sum_{n=0}^{N-1}\left[\alpha^{n} c\left(X_{n}, d_{n}\left(X_{n}\right)\right)\right], \\
w^{\pi}(x) & :=\limsup _{N \rightarrow \infty} \frac{1}{N} \mathbb{E}_{x}^{\pi} \sum_{n=0}^{N-1}\left[c\left(X_{n}, d_{n}\left(X_{n}\right)\right)\right],
\end{aligned}
$$

where $\alpha \in[0,1)$. Equations (II.1) and (II.2) define the infinite horizon expected discounted cost and the long-run average expected cost, respectively. In each case, define the optimal cost

$$
\begin{aligned}
v_{\alpha}(x) & :=\inf _{\phi \in \Pi} v_{\alpha}^{\phi}(x), \\
w(x) & :=\inf _{\phi \in \Pi} w^{\phi}(x),
\end{aligned}
$$

where $\Pi$ is the set of all policies. A policy $\phi$ is called optimal for the respective criterion if its value, $v_{\alpha}^{\phi}(x)$ or $w^{\phi}(x)$, corresponds to the value on the right hand side of (II.3) or (II.4) for all $x \in \mathbb{X}$. One goal of this research is to find conditions under which an optimal stationary policy exists.

It is well known (cf. [10, Proposition 9.8]) that $v_{\alpha}(x)$ satisfies the following discount cost optimality equations (DCOE),

$$
v_{\alpha}(x)=\inf _{a \in A(x)}\left\{c(x, a)+\alpha \int v_{\alpha}(y) q(d y \mid x, a)\right\},
$$

for $x \in \mathbb{X}$. In addition, if a stationary policy $\phi_{\alpha}$ satisfies the following equations for all $x \in X$

$$
v_{\alpha}(x)=c\left(x, \phi_{\alpha}(x)\right)+\alpha \int v_{\alpha}(y) q\left(d y \mid x, \phi_{\alpha}(x)\right),
$$

for $x \in \mathbb{X}$, then $\phi_{\alpha}$ is optimal. According to [1, Proposition 2.1], conditions (W) and (S), explained in the following section, imply the existence of a stationary policy that satisfies (II.6).

For the remainder of the paper, assume that the following condition holds.

Assumption (G): There exists a policy $\pi$ and an initial state $x$ such that

$$
w^{\pi}(x)<\infty .
$$

Note that this is equivalent to the General Assumption of Schäl [1]: $\inf _{x \in \mathbb{X}} \inf _{\phi \in \Pi} w^{\phi}(x)<\infty$.

Define the following quantities

$$
m_{\alpha}:=\inf _{x \in \mathbb{X}} v_{\alpha}(x), \quad u_{\alpha}(x):=v_{\alpha}(x)-m_{\alpha},
$$


and

$$
w^{*}=\liminf _{\alpha \rightarrow 1}(1-\alpha) m_{\alpha} .
$$

Assumption (G) implies that $w^{*}<\infty$; Schäl [1, Lemma 1.2]. According to Schäl [1, Proposition 1.3], if there exists a measurable function $u: \mathbb{X} \rightarrow[0, \infty)$ and a stationary policy $\phi$ such that

$$
w^{*}+u(x) \geq c(x, \phi(x))+\int u(y) q(d y \mid x, \phi(x)),
$$

for $x \in \mathbb{X}$, then the stationary policy $\phi$ is average cost optimal for and $w(x)=w^{*}$ for all $x \in \mathbb{X}$.

Schäl [1] describes conditions to guarantee that (II.9) holds for problems with compact action sets. We study the validity of (II.9) for problems with possibly non-compact action sets. The following condition plays an important role for the validity of (II.9).

Assumptions (B): Assumption (G) holds and $\sup _{\alpha<1} u_{\alpha}(x)<\infty$ for all $x \in \mathbb{X}$.

\section{KNOWN RESULTS}

In this section, we discuss some closely related results. Schäl [1] studies problems with compact action sets when transition probabilities satisfy either Assumptions (W) below; when the transition probabilities are weakly continuous or Assumptions (S) below; when the transition probabilities are setwise continuous. Each of these conditions together with (B) yield the existence of an optimal policy satisfying (II.9) and the convergence along a subsequence of both the optimal discounted cost and policies to those in the average cost case. By contrast, Hernandez-Lerma and Lasserre [8, section 5.4] do not require a compact action space, but deal only with MDPs whose transition probabilities are setwise continuous.

Let $\mathbf{C}(\mathbb{A})$ denote the set of all non-empty compact subsets of $\mathbb{A}$ and let $\mathbb{P}(\mathbb{X})$ be the set of all probability measures on $\mathbb{X}$. The following are two sets of assumptions are defined in Schäl [1].

\section{Assumptions (W):}

$0) \mathbb{X}$ is locally compact with a countable base,

1) $A(x) \in \mathbf{C}(\mathbb{A})$ for $x \in \mathbb{X}$,

2) $c$ is lower semicontinuous on $\operatorname{Gr}(\mathbb{A})$,

3) $A: \mathbb{X} \rightarrow \mathbf{C}(\mathbb{A})$ is upper semicontinuous, and

4) $q: \operatorname{Gr}(\mathbb{A}) \rightarrow \mathbb{P}(\mathbb{X})$ is continuous with respect to weak convergence on $\mathbb{P}(\mathbb{X})$.

\section{Assumptions (S):}

1) $A(x) \in \mathbf{C}(\mathbb{A})$ for $x \in \mathbb{X}$,

2) $c(x, \cdot): A(x) \rightarrow[0, \infty]$ is lower semicontinuous in $a$, and

3) $q(x, \cdot): A(x) \rightarrow \mathbb{P}(\mathbb{X})$ is continuous with respect to setwise convergence on $\mathbb{P}(\mathbb{X})$.

We next state the main results proved in Schäl [1, Proposition 3.5, Theorem 3.8].
Theorem 3.1: Let $(\mathbf{B})$ and either $(\mathbf{W})$ or $(\mathbf{S})$ hold. Then there exists a function $u: \mathbb{X} \rightarrow[0, \infty)$ and a stationary policy $\phi$ satisfying (II.9). Therefore, $w^{\phi}(x)=w(x)=w^{*}$. Furthermore $w^{*}=\lim _{\alpha \rightarrow 1}(1-\alpha) m_{\alpha}=\lim _{\alpha \rightarrow 1}(1-$ $\alpha) v_{\alpha}(x), x \in \mathbb{X}$. Fix a sequence of discount factors $\alpha(k) \rightarrow$ 1 .

1) Under (W),

a) The function $u$ can be defined

$$
u(x)=\liminf _{k \rightarrow \infty, y \rightarrow x} u_{\alpha(k)}(y) .
$$

b) For each $x \in \mathbb{X}$ there exists a subsequence $\alpha_{m}$ of the sequence $\alpha(k)$ such that the mapping $\phi(x)=\lim _{m \rightarrow \infty} \phi_{\alpha_{m}}\left(x_{m}\right)$ defines a stationary policy that satisfies (II.9) with $u$ defined in (III.1).

2) Under (S),

a) The function $u$ can be defined as

$$
u(x)=\liminf _{k \rightarrow \infty} u_{\alpha(k)}(x) .
$$

b) For each $x \in \mathbb{X}$ there exists a subsequence $\alpha_{m}$ of the sequence $\alpha(k)$ such that the mapping $\phi(x)=\lim _{m \rightarrow \infty} \phi_{\alpha_{m}}\left(x_{m}\right)$ defines a stationary policy satisfying (II.9) with $u$ defined in (III.2).

We remark that under Assumptions (W) the function $u$ defined in (III.1) is lower semicontinuous and under Assumptions (S) the function $u$ defined in (III.2) is measurable.

Definition 3.2: A real-valued function $f$ defined on a metric space $\mathbb{Y}$ is called inf-compact if for all $\lambda \in \mathbb{R}$ the sets $D(\lambda)=\{y \in \mathbb{Y} \mid f(y) \leq \lambda\}$ are compact.

The following appear as Assumptions 4.2.1 in HernandezLerma and Lasserre [8].

\section{Assumptions (HL):}

1) $c$ is inf-compact on $\operatorname{Gr}(\mathbb{A})$.

2) $q: G r(\mathbb{A}) \rightarrow \mathbb{P}(\mathbb{X})$ is setwise continuous, i.e.,

$$
f(x, a)=\int_{\mathbb{X}} g(y) q(y \mid x, a)
$$

is continuous and bounded whenever $\mathrm{g}$ is measurable and bounded.

We remark that if (II.9) holds for a stationary policy $\phi$ and for a non-negative function $u$ then

$$
w^{*}+u(x) \geq \inf _{a \in A(x)}\left\{c(x, a)+\int u(y) q(d y \mid x, a)\right\}
$$

for $x \in \mathbb{X}$, and each of the Assumptions (W), (S), and (HL) implies by The Arsenin-Kunugui Theorem [11, Theorem 35.46] the existence of a policy $\phi$ such that the minimum in (III.3) is achieved when $a=\phi(x)$. Thus, infimum can be replaced with minimum in (III.3). Next we formulate the results from [8] relevant to Theorem 3.1 .

Theorem 3.3: (see [8, Theorems 5.4.3 and 5.4.6]) Let (B) and (HL) hold. Then there exists a function $u: \mathbb{X} \rightarrow$ 
$[0, \infty)$ and a stationary policy $\phi$ satisfying (II.9). Therefore, $w^{\phi}(x)=w(x)=w^{*}$. Consider a sequence $\alpha(n) \rightarrow 1$ such that $\lim _{n \rightarrow \infty}(1-\alpha(n)) v_{\alpha(n)}(x)$ exists for some $x \in \mathbb{X}$ (such a sequence exists in light of the Lemma on p. 88 of [8]) Then for all $x \in \mathbb{X}$ this limit exists and equals $w^{*}$. Furthermore, the function $u$ can be defined as

$$
u(x)=\liminf _{n \rightarrow \infty} u_{\alpha(n)}(x) .
$$

\section{Main Structural Results}

We begin by stating assumptions for non-compact action sets; Assumptions (Wu) and (Su) below. These assumptions are similar to Assumptions (W) and (S), respectively. The letter "u" signifies unbounded action sets. In the interest of brevity, the proofs in this section are omitted.

\section{Assumptions (Wu):}

0) $\mathbb{X}$ is locally compact with a countable base,

1) $c$ is inf-compact on $\operatorname{Gr}(\mathbb{A})$, and

2) $q: \operatorname{Gr}(\mathbb{A}) \rightarrow \mathbb{P}(\mathbb{X})$ is continuous with respect to weak convergence on $\mathbb{P}(\mathbb{X})$.

\section{Assumptions (Su):}

1) $c$ is inf-compact on $A(x)$, and

2) $q(x, \cdot): A(x) \rightarrow \mathbb{P}(\mathbb{X})$ is continuous with respect to setwise convergence on $\mathbb{P}(\mathbb{X})$.

We recall that a stationary policy is optimal for the total expected discounted cost criteria if and only if (II.6) holds; see e.g. [12, Section 6.3]. The following proposition is similar to [1, Proposition 2.1] for models with compact action sets.

Proposition 4.1: Under (Wu) or $(\mathbf{S u})$ there exists a stationary, discounted cost optimal policy $\phi_{\alpha}$; i.e. (II.6) holds for $\phi_{\alpha}$. Moreover, the value function $v_{\alpha}$ is inf-compact under $(\mathbf{W u})$ and measurable under $(\mathbf{S u})$.

The next definition is an extension of that of locally bounded functions on $\mathbb{R}^{n}$ (cf. p. 113 of [13]).

Definition 4.2: A real-valued function $f$ defined on a metric space $\mathbb{Y}$ is called locally bounded above at $x \in \mathbb{Y}$ if there exists an open set $B(x)$ containing $x$ such that

$$
\sup _{y \in B(x)} f(y)<\infty
$$

The function $f$ is called locally bounded above if it is locally bounded for each $x \in \mathbb{Y}$.

For a point $x \in \mathbb{Y}$, let $\mathcal{O}(x):=\{B \subseteq Y \mid x \in$ $B, B$ is open $\}$ be the set of open sets containing $x$. Consider the function

$$
\bar{f}(x):=\inf _{B \in \mathcal{O}(x)} \sup _{y \in B} f(y) .
$$

Obviously, if $f$ is locally bounded at $x, f(x) \leq \bar{f}(x)<\infty$.

Lemma 4.3: Suppose $f$ is a locally bounded above function on a complete, separable, metric space $\mathbb{Y}$. Then the function $\bar{f}(x)$ is upper semicontinuous.

For $\alpha \in[0,1)$ define $r_{\alpha}(x)=\sup _{\alpha \leq \beta<1} u_{\beta}(x)$. Assumptions (B) are equivalent to the validity of the following pair of assumptions: (i) Assumption (G), and (ii) for any $x \in \mathbb{X}$ there exists $\alpha \in[0,1)$ with $r_{\alpha}(x)<\infty$. Thus, (B) are equivalent to [8, Condition 5.4.5] and imply that $r_{\alpha}(x)<\infty$ for all $\alpha \in[0,1)$ and for $x \in \mathbb{X}$.

Let $\Gamma_{\alpha}(x):=\sup _{\alpha \leq \beta<1}\left\{v_{\beta}(x)-\beta m_{\beta}\right\}$, where $\alpha \in$ $[0,1)$. According to [1, Lemma 1.2], Assumption (G) implies that $\lim \sup _{\alpha \rightarrow \infty}(1-\alpha) m_{\alpha}<\infty$. Thus, if (B) hold, there exists $\alpha^{*} \in[0,1)$ such that for all $\alpha \in\left[\alpha^{*}, 1\right)$

$$
\Gamma_{\alpha}(x) \leq r_{\alpha}(x)+\sup _{\alpha \leq \beta<1}\left\{(1-\beta) m_{\beta}\right\}<\infty .
$$

The following condition strengthens (B).

Assumptions (LB). Assumption (G) holds and there exists $\alpha_{0} \in[0,1)$ such that the function $r_{\alpha_{0}}(x)$ is locally bounded above on $\mathbb{X}$.

We observe that the function $\Gamma_{\alpha}(x)$ is decreasing in $\alpha$. Thus, if (LB) hold then the function $\Gamma_{\alpha}$ is locally bounded for any $\alpha \in\left[\alpha_{0}, 1\right)$. Select an arbitrary $\alpha^{*} \in\left[\alpha_{0}, 1\right)$ such that (IV.1) holds when $\alpha=\alpha^{*}$ and denote $\Gamma=\Gamma_{\alpha^{*}}$.

We say that an MDP is a submodel of another MDP if the only difference between these MDPs is that the sets of available actions of the former MDP are subsets of available actions of the latter MDP. Define the following subsets of the set of available actions in state $x$

$$
\tilde{A}(x):=\{a \in A(x) \mid c(x, a) \leq \bar{\Gamma}(x)\} .
$$

Let Assumptions (LB) hold and $\alpha \in\left[\alpha_{0}, 1\right)$. According to Lemma 4.3, the function $\bar{\Gamma}$ is upper semicontinuous and is therefore measurable. This implies that the graph of the mapping $\tilde{A}: \mathbb{X} \rightarrow \mathbb{A}$ is Borel, and the set $\{\mathbb{X}, \mathbb{A}, \tilde{A}(x), q, c\}$ defines a submodel of the original $\operatorname{MDP}\{\mathbb{X}, \mathbb{A}, A(x), q, c\}$.

Definition 4.4: We say that a submodel is equivalent to the original MDP if there exists $\tilde{\alpha} \in[0,1)$ such that, under the $\alpha$-discounted cost criterion with $\alpha \in[\tilde{\alpha}, 1)$, a stationary policy is optimal for the submodel if and only if it is optimal for the original MDP.

Proposition 4.5: (i) If the original MDP satisfies Assumptions (LB) and (Wu) or (Su) then the submodel $\{\mathbb{X}, \mathbb{A}, \tilde{A}(x), q, c\}$ is equivalent to the original MDP and this submodel satisfies Assumptions (B) and (W) or (S), respectively.

Proposition 4.5 and Theorem 3.1 imply the main result of this section.

Theorem 4.6: The statement of Theorem 3.1 remains valid with the Assumptions (B), (W), and (S) substituted with Assumptions (LB), (Wu), and (Su) respectively. In addition, the function $u$ is lower semicontinuous under (Wu) and is measurable under (Su).

In the conclusion of this section, we make the following remarks on the average cost optimality inequalities. As was explained above (see the paragraph containing (III.3)), the assumptions of Theorems 3.1, 3.3, and 4.6 imply the existence of a function $u: \mathbb{X} \rightarrow[0, \infty)$ and a stationary 
policy $\phi$ such that

$$
\begin{aligned}
w^{*}+u(x) & \geq \min _{a \in A(x)}\left\{c(x, a)+\int u(y) q(d y \mid x, a)\right\} \\
& =c(x, \phi(x))+\int u(y) q(d y \mid x, \phi(x)) .
\end{aligned}
$$

In particular, under the assumptions of Theorem 3.3 a stationary policy that satisfies (IV.2), was constructed in [8, p. 90]. Cavazos-Cadena [14] discuss an important example where (IV.2) holds for a stationary policy $\phi$ but the version of these formulae with the equality, replacing the inequality, does not hold because there is strict inequality in at least one state. We recall that [14, Example 3.1] consists of two policies, $\phi$ and $f$. The policy $f$ from [14] satisfies (II.9) but it does not satisfy (IV.2).

\section{Applications to InVEntory Control}

We begin by considering the classic inventory control model with a fixed ordering cost. This is then extended to the cash balance problem. As is the case in Ye and Duenyas [15] we define a (K,Q)-convex function.

Definition 5.1: A real-valued function $f$ is called $(K, Q)-$ convex if for any $x \leq y, K, Q \geq 0$ and $\lambda \in[0,1]$,

$$
\begin{aligned}
f((1-\lambda) x+\lambda y) \leq & (1-\lambda) f(x)+\lambda f(y)+\lambda K \\
& +(1-\lambda) Q \\
& -\min \{\lambda, 1-\lambda\} \min \{K, Q\} .
\end{aligned}
$$

A function is called $(K, Q)$-concave if $-f$ is $(K, Q)$-convex. A $(K, 0)$-convex function is called $K$-convex.

\section{A. Inventory Control with a Fixed Ordering Cost}

Consider a single commodity inventory system where in each period there is the possibility of demand for inventory on hand. A decision-maker must decide how much of the product to order to meet demand. Assume the unmet demand is backlogged and the cost of inventory held or backlogged (negative inventory) is modeled as a convex function. The objective is to minimize the expected discounted or average cost over an infinite horizon. Let

- $\alpha \in(0,1)$ be the discount factor,

- $K \geq 0$ be a fixed production cost,

- $c>0$ be the per unit ordering cost,

- $h(\cdot)$ denote the holding/backordering cost per period; convex, non-negative, takes finite values, and $h(x) \rightarrow$ $\infty$ as $|x| \rightarrow \infty$,

- $\left\{D_{n}, n \geq 0\right\}$ be a sequence of i.i.d. random variables where $D_{n} \geq 0$ (almost surely) represents demand in the $\mathrm{n}^{\text {th }}$ period. We assume that $\mathbb{E} h(y-D)<\infty$ for all $y \in \mathbb{R}$, where $D$ is a random variable with the same distribution as $D_{n}$. We notice, that this assumption and the assumed properties of the function $h$ imply that $\mathbb{E}|D|<\infty$.
One should make the important observation that we have only made light assumptions on the demand distribution. That is to say, that unlike all of the literature we found we need not restrict attention to continuous or discrete distributions or even to those that have densities.

In keeping with classic results, we expect that there exists inventory levels $s$ and $S$ such that if the inventory level falls below $s$, the manager should order up to level $S$, otherwise no ordering should be done. The difference between the two levels is due to the fact that the fixed cost gives an incentive to order less often.

Lemma 5.2: In the inventory control model, Assumptions (LB) and (W) hold. Moreover, there exists $\alpha^{*}$ such that function $v_{\alpha}$ is $K-$ convex and continuous for all $\alpha \in\left[\alpha^{*}, 1\right)$.

This leads to the main theorem of this section.

Theorem 5.3: There exists thresholds $s$ and $S$ such that the following order-up-to policy is average cost optimal.

$$
t^{\prime}(y)= \begin{cases}S & \text { if } y \leq s \\ y & \text { if } y>s\end{cases}
$$

That is, there exists average cost optimal $(s, S)$ policies.

\section{B. The Cash Balance Problem with Fixed Costs}

The cash balance problem is an extension of the previous inventory problem. The requirement that demand is nonnegative is relaxed and the decision-maker can choose to increase inventory via production or decrease it via scrapping. This scenario models not only the decision of how much cash to hold on-hand for a firm's financial transactions, but also product returns in inventory control. Classic work on this problem was done by Eppen and Fama [16] for the problem without fixed costs and by Girgis [17] and Neave [18] for a single fixed cost (either ordering or scrapping) and with multiple fixed costs, respectively. More recent work to simplify these proofs can be found in Chen and Simchi-Levi [19] and extensions in the case without fixed costs in Feinberg and Lewis [20].

Assume that all of the quantities defined in the previous section remain in place and add the following

- $Q \geq 0$ is the fixed scrapping cost

- $c_{-}>0$ is the per unit scrapping cost.

Without loss of generality assume that $K \geq Q$. Moreover, relax the assumption that the per period demand $D$ must be non-negative. We assume only that $P(D \neq 0)>0$ and $\mathbb{E} h(x-D)<\infty$ for any $x \in \mathbb{R}$. For consistency we add a superscript to the per unit ordering cost and denote it $c_{+}$. As is the case in [18] and [19], assume the existence of $x \leq y \leq w \leq z$ such that

$$
\begin{aligned}
& \frac{\mathbb{E}[h(y-D)-h(x-D)]}{y-x}<-c^{+}, \\
& \frac{\mathbb{E}[h(z-D)-h(w-D)]}{z-w}>c_{-} .
\end{aligned}
$$


Lemma 5.4: Assumption (LB) and (W) hold and the optimal discounted cost value function is $(\mathrm{K}, \mathrm{Q})$-convex and continuous in the inventory level.

This leads to the main result of this section.

Theorem 5.5: There exists scalars $S, s, s^{\prime}, u^{\prime}, u$, and $U$ such that the following policy is average cost optimal. If $K>Q$,

$$
t^{\prime}(y)= \begin{cases}S & \text { if } y \leq s, \\ \in\{y, S\} & \text { if } y \in\left(s, s^{\prime}\right), \\ y & \text { if } y \in\left[s^{\prime}, u^{\prime}\right), \\ \in\left[s^{\prime}, y\right] & \text { if } y \in\left[u^{\prime}, u\right), \\ U & \text { if } y \geq u .\end{cases}
$$

If $K=Q$,

$$
t^{\prime}(y)= \begin{cases}S & \text { if } y \leq s, \\ \in\{y, S\} & \text { if } y \in\left(s, \frac{s+S}{2}\right), \\ y & \text { if } y \in\left(\frac{s+S}{2}, \frac{u+U}{2}\right), \\ \in\{y, U\} & \text { if } y \in\left(\frac{u+U}{2}, u\right), \\ U & \text { if } y \geq u .\end{cases}
$$

\section{CONCLusions}

In this paper we presented two sets of conditions that lead to the convergence of the optimal discounted cost value function and policies to those in the average cost case. In each case, the results of Schäl [1] serve as the basis for the analysis, but inf-compactness and local boundedness conditions replace the compact action set assumption in Schäl [1]. We then apply the results to the classic inventory and cash balance models. An interesting question that needs to be answered is which of the previous computational results (cf. [6], [4], [21]) extend when the demand distribution is more general? A second possibility is to extend this work to other recent work on combined pricing and inventory decisions (cf. [22], [23]). We leave these as potential future research directions.

\section{ACKNOWLEDGEMENTS}

We would also like to thank Irwin Schochetman for providing several references for upper semicontinuity of a set-valued function. Research of the first author was partially supported by NSF grant DMI-0300121.

\section{REFERENCES}

[1] M. Schäl, "Average optimality in dynammic programming with general state space," Math. Oper. Res., vol. 18, pp. 163-172, 1993.

[2] E. Porteus, "Stochastic inventory theory," in Handbooks in Operations Research and Management Science, D. Heyman and M. Sobel, Eds. Amsterdam: Elsevier Science Publishers, 1990, vol. 2, ch. 12, pp. 605-652.
[3] D. L. Iglehart, Dynamic Programming and Stationary Analysis of Inventory Problems, ser. Office of Naval Research Monographs on Mathematical Methods in Logistics. Stanford, California: Stanford University Press, 1963, ch. 1, pp. 1-31.

[4] A. F. Veinott, Jr. and H. M. Wagner, "Computing optimal $(\mathrm{s}, \mathrm{S})$ policies," Management Science, vol. 11, no. 5, pp. 525-552, March 1965.

[5] Y. S. Zheng, "A simple proof for the optimality of $(\mathrm{s}, \mathrm{S})$ policies in infinite horizon inventory systems," Journal of Applied Probability, vol. 28, pp. 802-810, 1991.

[6] D. Beyer and S. Sethi, "The classical average-cost inventory models of Iglehart and Veinott-Wagner revisited," Journal of Optimization and Applications, vol. 101, pp. 523-555, 1999.

[7] L. I. Sennott, Stochastic Dynamic Programming and the Control of Queueing Systems. New York: John Wiley and Sons, Inc., 1999.

[8] O. Hernández-Lerma and J. B. Lasserre, Discrete-Time Markov Control Processes: Basic Optimality Criteria. New York, NY: Springer, 1996.

[9] E. Fernández-Gaucherand, A. Arapostathis, and S. I. Marcus, "Convex stochastic control problems," in Proceedings of the 31st Conference on Decision and Control. IEEE, December 1992, pp. 21792180.

[10] D. P. Bertsekas and S. E. Shreve, Stochastic Optimal Control: The Discrete-Time Case. Belmont, MA: Athena Scientific, 1996.

[11] A. S. Kechris, Classical descriptive set theory. New York: SpringerVerlag, 1994.

[12] E. A. Dynkin and A. A. Yushkevich, Controlled Markov Processes. New York: Springer-Verlag, 1979

[13] H. S. Gaskill and P. Narayanaswami, Elements of Real Analysis. Upper Saddle River, New Jersey: Prentice-Hall, 1998.

[14] R. Cavazos-Cadena, "A counterexample on the optimality equation in markov decision chains with the average cost criterion," Systems \& Control Letters, vol. 16, pp. 387-392, 1991.

[15] Q. Ye and I. Duenyas, "Optimal joint capacity investment and pricing/production quantity decisions with random demand and fixed capacity adjustment costs," 2004, preprint.

[16] G. D. Eppen and E. F. Fama, "Cash balance and simple dynamic portfolio problems with proportional costs," International Economic Review, vol. 10, no. 2, pp. 119-133, June 1969.

[17] N. M. Girgis, "Optimal cash balance levels," Management Science, vol. 15 , no. 3, pp. 130-140, November 1968.

[18] E. H. Neave, "The stochastic cash balance problem with fixed costs for increases and decreases," Manag. Sci., vol. 16, pp. 472-490, 1970.

[19] X. Chen and D. Simchi-Levi, "A new approach for the stochastic cash balance problem with fixed costs," 2004, preprint.

[20] E. A. Feinberg and M. E. Lewis, "Optimality of four-threshold policies in inventory systems with customer returns and borrowing/storage options," Probability in the Engineering and Informational Sciences, 2004, to appear.

[21] Y. S. Zheng and A. Federgruen, "Finding optimal $(\mathrm{s}, \mathrm{S})$ policies is about as simple as evaluating a single policy," Operations Research, vol. 39, no. 4, pp. 654-665, July-August 1991.

[22] X. Chen and D. Simchi-Levi, "Coordinating inventory control and pricing strategies with random demand and fixed ordering cost: The infinite horizon case," Math. Oper. Res., vol. 29, pp. 698-723, 2004.

[23] — - "Coordinating inventory control and pricing strategies with random demand and fixed ordering cost: The finite horizon case," Operations Research, 2004, to appear. 